\title{
Editorial
}

\section{Computer Virus: Theory, Model, and Methods}

\author{
Xiaofan Yang, ${ }^{1}$ Bimal Kumar Mishra, ${ }^{2}$ and Yanbing Liu ${ }^{3}$ \\ ${ }^{1}$ School of Electronic and Information Engineering, Southwest University, Chongqing 400715, China \\ ${ }^{2}$ Department of Applied Mathematics, Birla Institute of Technology, Mesra, Ranchi 835215, India \\ ${ }^{3}$ School of Communication and Information Engineering, Chongqing University of Posts and \\ Telecommunications, Chongqing 400065, China \\ Correspondence should be addressed to Xiaofan Yang, xfyang1964@gmail.com
}

Received 22 November 2012; Accepted 22 November 2012

Copyright (c) 2012 Xiaofan Yang et al. This is an open access article distributed under the Creative Commons Attribution License, which permits unrestricted use, distribution, and reproduction in any medium, provided the original work is properly cited.

Computer viruses are a large class of malicious codes that can spread among computers and perform detrimental operations. This issue on theory, model, and methods of computer virus aims to better understand the way computer viruses spread on the Internet and thereby work out effective policies of defending against them. This issue contains 12 fascinating papers, with a focus on epidemic modeling.

The majority of previous computer virus epidemic models neglect the notable difference between computer viruses and infectious diseases. Noting that all infected computers have infectivity naturally, X. Yang and L.-X. Yang suggest a series of novel epidemic models, known as the SLBS models. Toward this direction, L.-X. Yang and X. Yang address a special SLBS model, declaring its global dynamics. By incorporating a temporary immunity compartment in an SLBS model, M. Yang et al. derive a new model, known as the SLBRS model, revealing its global behaviors.

In reality, virus spreading is influenced by multifarious factors. C. Gan et al. examine the effect of human intervention on virus spreading, proclaiming the global properties of the proposed model. J. Ren et al. investigate the influence of time-varying delay on virus spreading, obtaining similar theoretical results. In view of impulsive vaccinations, C. Zhang et al. devise an impulsive epidemic model, arguing the existence of a globally attractive periodic solution provided the vaccination rate is large enough. Noting the inevitable fluctuation of a system parameter, C. Zhang et al. induce a stochastic epidemic model, announcing its global stability.

As autonomous computer viruses, worms and botnets turn out to be a great threat to information security. By incorporating three additional compartments in the conventional SEIS model, Yao et al. propose a delayed epidemic model of worms, showing that a Hopf bifurcation phenomenon might occur. L. Feng et al. raise a model of peer-to-peer botnet and investigate its global dynamics from both the microlevel and the macrolevel. 
Smart mobile terminals have come to be an integral part of the Internet. By a close inspection of the features of both smart mobile terminals and human behaviors, Y. Liu et al. propose a practical virus detection scheme.

The Internet turns out to possess scale-free property, with node degrees following a power-law distribution. In this context, C.-Y. Huang and C.-T. Sun probe deeply the effect of available resources and costs on the spread of computer viruses.

Xiaofan Yang

Bimal Kumar Mishra

Yanbing Liu 


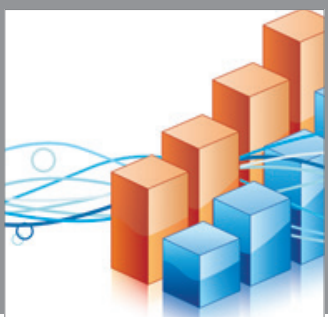

Advances in

Operations Research

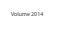

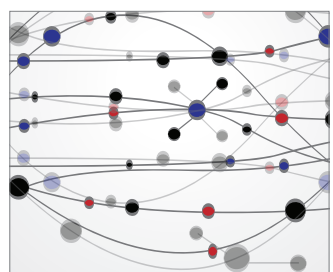

\section{The Scientific} World Journal
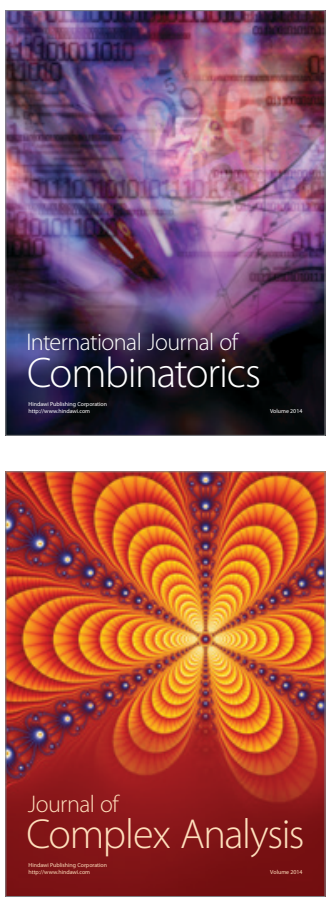

International Journal of

Mathematics and

Mathematical

Sciences
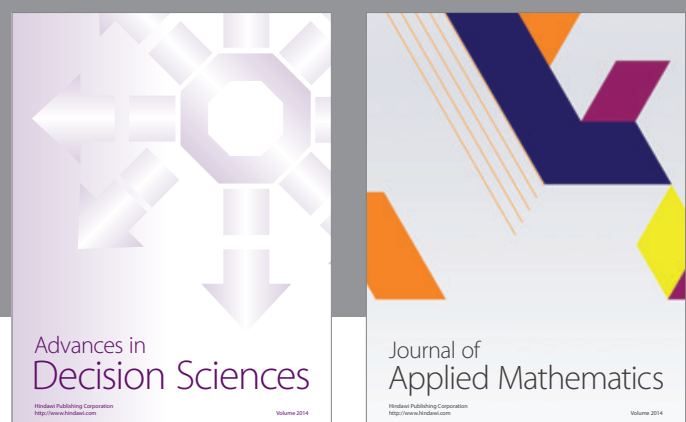

Journal of

Applied Mathematics
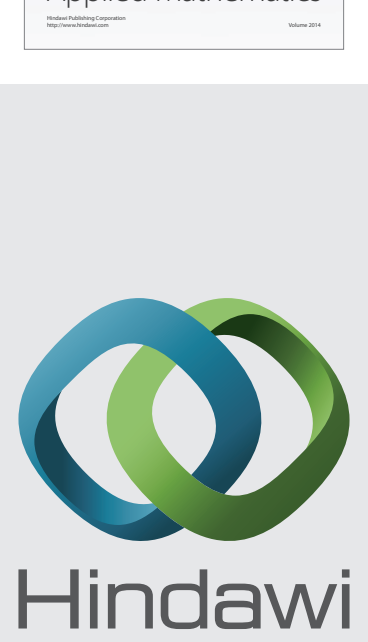

Submit your manuscripts at http://www.hindawi.com
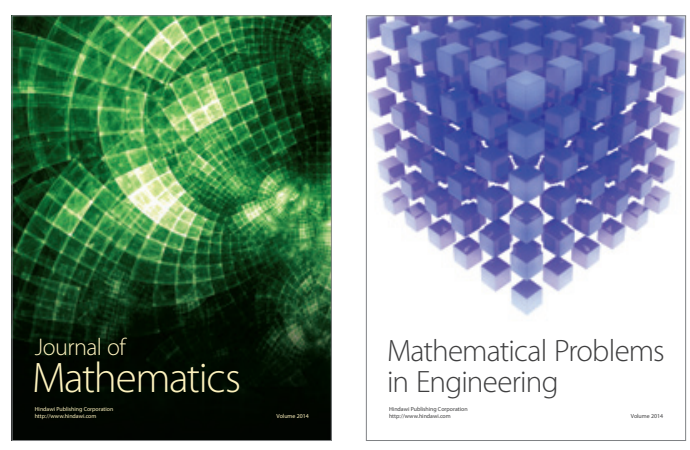

Mathematical Problems in Engineering
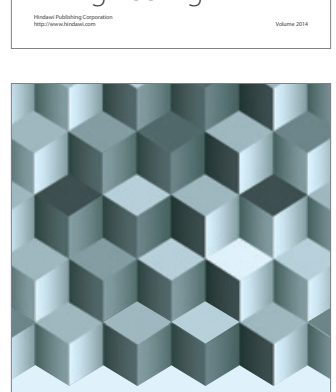

Journal of

Function Spaces
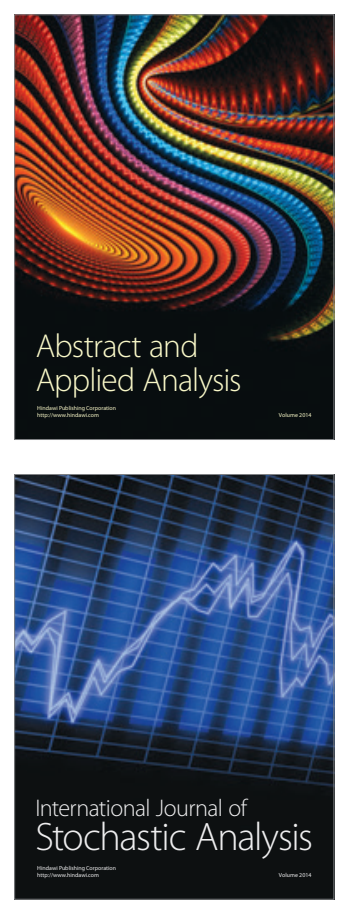

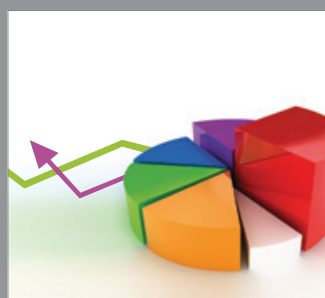

ournal of

Probability and Statistics

Promensencen
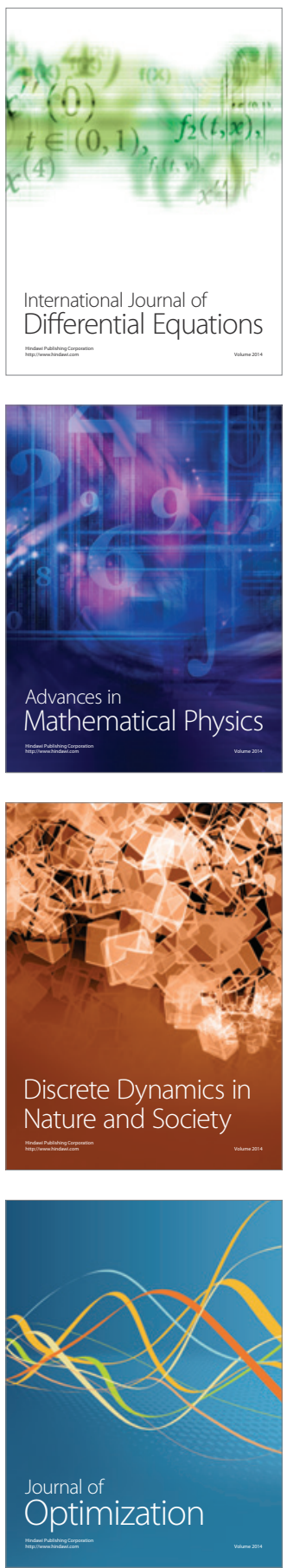